\title{
Immersive Brain Puzzle: aplicação de realidade virtual voltada à reabilitação de pacientes pós-AVC
}

\author{
Juliana de Fátima Ovídio Araújo \\ Departamento de Ciência da Computação - DCOMP \\ Universidade Federal de São João del-Rei - UFSJ \\ São João del-Rei, Brasil \\ julianadft@gmail.com
}

\author{
Alexandre Fonseca Brandão \\ Instituto de Física "Gleb Wataghin- IFGW \\ Universidade Estadual de Campinas - Unicamp \\ Campinas, Brasil \\ brandaobiotec@gmail.com
}

\author{
Diego Roberto Colombo Dias \\ Departamento de Ciência da Computação - DCOMP \\ Universidade Federal de São João del-Rei - UFSJ \\ São João del-Rei, Brasil \\ diegodias@ufsj.edu.br
}

\begin{abstract}
Resumo-A aplicação Immersive Brain Puzzle tem o intuito de auxiliar o processo de reabilitação de pacientes de acidente vascular cerebral (AVC). Geralmente, no início do tratamento, quando o paciente ainda está na unidade de tratamento intensivo (UTI), o processo de reabilitação é muito restrito, visto que o ambiente de uma UTI não é propício para este tipo de atividade. No entanto, estudos apontam que as primeiras horas após um AVC são importantes para o tratamento dos pacientes. Deste modo, essa iniciação científica aborda a proposta e implementação de uma aplicação que permita que o paciente que sofreu um AVC possa iniciar sua atividade de reabilitação mesmo na UTI, por meio de um ambiente imersivo e interativo, que visa estimular o processo motor e cognitivo. A aplicação já foi desenvolvida, de modo que a próxima etapa será a validação, realizada junto ao Brazilian Institute of Neuroscience and Neurotechnology (BRAINN) - Unicamp.

Resumo-The Immersive Brain Puzzle application is intended to assist the rehabilitation process of stroke patients. Generally, at the beginning of treatment, when the patient is still in the intensive care unit (ICU), the rehabilitation process is very restricted, since the ICU environment is not a suitable place for this type of activity. However, studies indicate that the first hours after a stroke are important for the treatment of patients. Thus, this scientific initiation addresses the proposal and implementation of an application that allows the patient, who has suffered a stroke, can start their rehabilitation activity even in the ICU, through an immersive and interactive environment, which aims to stimulate the motor and cognitive processes. The application has already been developed, so the next step will be validation, performed with the Brazilian Institute of Neuroscience and Neurotechnology (BRAINN) - Unicamp.
\end{abstract}

Index Terms-realidade virtual, reabilitação, acidente vascular cerebral

\section{INTRODUÇÃO}

O Acidente Vascular Cerebral (AVC), também conhecido como Acidente Vascular Encefálico (AVE) ou "Derrame Cerebral"ocorre quando o suprimento de sangue que vai para o cérebro é interrompido ou drasticamente reduzido, privando as cédulas de oxigênio e de nutrientes. Pode ainda ocorrer quando um vaso sanguíneo se rompe, causando uma hemorragia cerebral [5].

O AVC é doença cerebrovascular mais prevalente na população idosa, apesar de acometer todas as faixas etárias. Ademais, ocupa a terceira causa de morte nos países desenvolvidos, superada apenas pelas doenças coronarianas e o câncer. De acordo com Organização Mundial de Saúde, o Brasil é o sexto na lista dos países com maior número de vítimas de AVC, precedido apenas pela China, Índia, Rússia, EUA e Japão [7].

Diversas modalidades terapêuticas são disponíveis para a reabilitação de pacientes com AVC, tais como terapia por contensão induzida e terapia orientada à tarefa, capazes de auxiliar na restauração das funções afetadas [1]. No entanto, tais recursos podem tornar-se desmotivadores e monótonos ao paciente em função da repetição intensiva. Nesse contexto, surgiu a terapia por meio da Realidade Virtual (RV), composta por exercícios baseados em jogos e aplicações virtuais, que contribuem de maneira lúdica para a facilitação do movimento normal e treinamento funcional [8].

Estudos da literatura acerca do tema concluíram que a utilização da RV tem se mostrado efetiva no tratamento de pacientes que sofreram AVC. Entretanto, ressaltam que devido ao fato da metodologia ser recente, é necessário um maior número de casos de estudo. [6].

Neste trabalho é apresentada aplicação Immersive Brain Puzzle, desenvolvida com intuito de auxiliar o processo de reabilitação de pacientes que sofreram AVC, apresentando-lhes um jogo de quebra-cabeça em realidade virtual. Ademais, é possível que o profissional responsável realize o acompanhamento do progresso do paciente por meio de uma aplicação em um dispositivo independente, permitindo-lhe a visualização do jogo em tempo real.

A pesquisa ainda está em condução, uma vez que aplicação foi finalizada no início de julho. A próxima etapa consiste em realizar a avaliação da aplicação junto ao Brazilian Institute of Neuroscience and Neurotechnology (BRAINN), grupo de 
pesquisa interdisciplinar, com foco em pesquisas voltadas ao entendimento do cérebro humano, explorando áreas como: genética, neurobiologia, farmacologia, neuroimagem, ciência da computação, robótica, física e engenharia.

\section{TRABALHOS CORRELATOS}

Nesta seção são apresentados alguns trabalhos correlatos à pesquisa, que também aplicam RV à reabilitação. Os trabalhos selecionados avaliam os efeitos da RV em pacientes pósAVC, comparando os resultados obtidos com os resultados de pacientes submetidos a terapias convencionais.

Mirelman et al. [4] realizaram um ensaio clínico randomizado para avaliar o efeito da RV na biomecânica da marcha de indivíduos com hemiparesia causada por acidente vascular cerebral. Os pacientes foram divididos randomicamente em dois grupos, ambos com nove indivíduos cada. Os participantes do grupo que utilizaram RV receberam um treino realizado a partir do sistema Rutgers de reabilitação do tornozelo (RARS), baseado em uma plataforma de força com um sistema de feedback e seis graus de liberdade de movimento. Os pacientes permaneciam sentados em frente à tela do computador com o pé afetado fixo sobre a plataforma de força, e realizavam movimentos de dorsiflexão, flexão plantar, inversão e eversão do tornozelo para controlar aviões e barcos virtuais projetados na tela de um computador. O grupo não-RV realizou exercícios similares, porém sem realidade virtual. $\mathrm{O}$ treinamento foi realizado três vezes por semana, durante 4 semanas, por aproximadamente uma hora cada. A avaliação cinemática da marcha foi obtida por meio de oito câmaras de captação de movimento (Sistema Vicon $®$ ). O estudo mostrou que o grupo RV apresentou aumento significativamente maior na geração de força no tornozelo, além de melhora nas amplitudes de movimento (19,5\%) em relação ao grupo não-RV (3,3\%). Nenhuma mudança significativa foi observada na cinemática ou cinética do quadril pós-treino.

Kim et al. [3] examinaram o efeito da RV por meio do sistema IREX na negligência espacial unilateral de pacientes que sofreram AVC, a fim de avaliar os efeitos da RV na perceção. Os participantes foram divididos randomicamente em dois grupos, compostos por 12 indivíduos cada. $\mathrm{O}$ grupo $\mathrm{RV}$ recebeu treinamento em realidade virtual e reabilitação cognitiva baseada em computador, enquanto o grupo que não utilizou RV recebeu apenas reabilitação cognitiva baseada em computador. Foram então utilizados os seguintes instrumentos de avaliação: teste neuropsicológico computadorizado e o teste Tower of London (TOL) para avaliação da função cognitiva, índice Barthel modificado coreano (K-MBI) para avaliação do estado funcional e o índice de motricidade (MI) para avaliação da função motora. Todos os pacientes foram submetidos a essas avaliações antes da reabilitação e quatro semanas após o processo. O estudo mostrou que ambos os grupos apresentaram melhoras significativas na negligência espacial unilateral e na realização de suas atividades de vida diária; porém, o grupo que utilizou RV apresentou melhora superior nos testes clínicos de heminegligência. Os autores acreditam que o treinamento em realidade virtual combinado com a reabilitação cognitiva baseada em computador pode ser um benefício adicional para o tratamento do comprometimento cognitivo em pacientes com AVC.

Por fim, Cameirão et al. [2] fizeram um estudo dos efeitos do Rehabilitation Gaming System (RGS) no membro superior de pacientes pós-AVC. O RGS consiste em vários elementos que permitem que os movimentos reais do braço rastreados sejam reconstruídos nos movimentos dos braços virtuais no sistema de RV. Além disso, o RGS permite diagnósticos rápidos e online das capacidades do paciente e a personalização dinâmica do cenário de reabilitação. Os pacientes foram divididos em dois grupos com oito indivíduos cada e realizaram o treino durante doze semanas. O primeiro grupo utilizou o RGS e terapia convencional, enquanto o segundo grupo realizou um tratamento alternativo, que consistiu em terapia ocupacional intensa ou jogos interativos inespecíficos. Ao final do tratamento, pôde ser observado que o grupo RGS apresentou desempenho significativamente melhor na velocidade do braço parético que foi acompanhado por um melhor desempenho na subparte do braço. Além disso, o grupo RGS apresentou uma melhora significativamente mais rápida ao longo do durante o período de tratamento.

\section{MATERIAIS E MÉTODOS}

A pesquisa está embasada em estudos realizados dentro do escopo do CEPID BRAINN, em que as hipóteses de pesquisa foram levantadas por pesquisadores de área de tecnologia e saúde. A principal hipótese pode ser descrita da seguinte forma: Existem benefícios no emprego de aplicações imersivas que estimulem o processo motor e cognitivo nas primeiras horas da reabilitação de pacientes que sofreram AVC?

A fim de comprovar a hipótese levantada, uma aplicação foi proposta, visto que devido às limitações impostas pelo ambiente (UTI), as soluções encontradas na literatura e as já desenvolvidas pelo CEPID BRAINN não satisfaziam os requisitos.

Para elencar as funcionalidades da aplicação, um levantamento de requisitos foi realizado junto aos médicos e fisioterapeutas do CEPID BRAINN. Foram levantados os requisitos funcionais e não-funcionais.

Os requisitos funcionais levantados foram:

- a aplicação deve representar as regras de um quebracabeça, contendo peças fora de ordem;

- o paciente deve ser capaz de visualizar um braço virtual dentro do ambiente;

- o dispositivo imersivo a ser utilizado deve ser o head mounted display (HMD), preferencialmente wireless;

- a câmera do dispositivo deve ser fixa, de modo que o movimento de cabeça do paciente não prejudique a visualização da tabuleiro;

- o dispositivo de interação deve ser baseado em sensores inerciais, de modo que o paciente possa realizar a interação por meio do movimento dos seus membros superiores, mesmo em situações de restrição de seus movimentos; 
- o profissional da área de saúde deve ser capaz de visualizar a aplicação com a qual o paciente interage, por meio de outro dispositivo (computador ou smartphone);

- o profissional da área de saúde deve ser capaz de interagir com o ambiente do paciente, a fim de realizar modificações no ambiente, tais como: alterar a configuração das peças, selecionar uma nova imagem para o quebracabeça, reposicionar o braço virtual do usuário dentro do ambiente, com o intuito de auxiliá-lo nas atividades; e

- a amplitude do movimento deve ser configurável, a fim de permitir a interação dos pacientes independente de suas limitações.

Os requisitos não-funcionais levantados foram:

- o tempo de resposta entre o output dos sensores e o input da aplicação deverá ser feita em tempo-real;

- considerando a grande heterogeneidade de usuários em relação a idade, desempenho cognitivo e interação prévia com ambientes imersivos, a aplicação deve possuir uma interface amigável; e

- a aplicação deve ser compatível com diversas plataformas, preferencialmente com o Android.

A solução de desenvolvimento adotada foi a Unity, devido a alta compatibilidade com diversos dispositivos, inclusive com o GearVR, dispositivo escolhido para ser utilizado neste trabalho. A Figura 1 apresenta o dispositivo selecionado.



Figura 1. GearVR

A aplicação consiste em um quebra-cabeça, onde uma imagem randômica é dividida em nove partes. Cada uma dessas partes pode sofrer uma rotação de $90^{\circ}, 180^{\circ}$ ou $270^{\circ}$. O objetivo é que o paciente as movimente de modo que sua configuração original seja alcançada. A abordagem de rotacionar as peças foi escolhida devido às limitações dos pacientes, visto que pessoas que sofreram um AVC tendem a ter seus movimentos restritos, ainda mais nas primeiras horas após o ocorrido. A Figura 2 apresenta as nove partes do quebra-cabeça com suas rotações embaralhadas, e, em seguida, sua configuração final obtida.

Para que possa haver uma supervisão, o jogo é composto por duas aplicações: uma utilizada pelo paciente e a outra pelo profissional de saúde. O intuito é que o profissional possa acompanhar e auxiliar o paciente de acordo com a
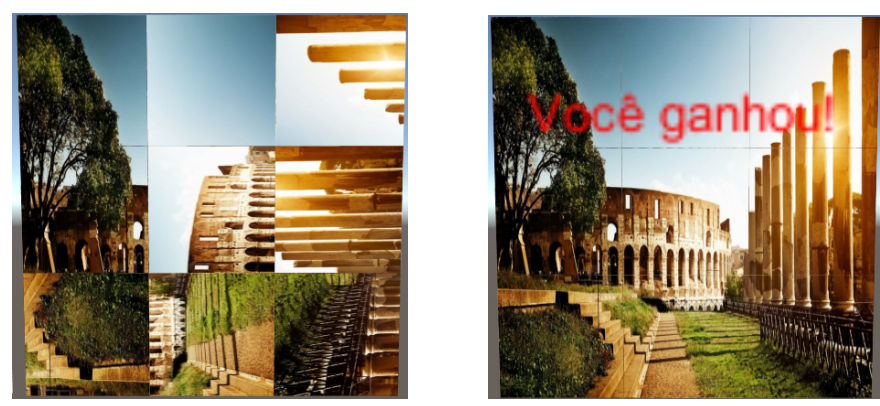

Figura 2. Exemplo de configuração inicial do jogo, seguido de sua configuração original

sua necessidade, além de realizar as configurações iniciais. A conexão das aplicações é realizada via rede, utilizando o NetworkManager do Unity.

O jogo é visualizado pelo paciente por meio de um HMD. Para a interação, foi utilizado um controle para apontar e rotacionar as peças. Não existe a necessidade de pegar e soltar a peça em razão das limitações de movimento que podem ser apresentadas. Deste modo, o paciente deve apenas posicionar a mão virtual sobre a peça que ele deseja rotacionar. Por meio de um clique, a peça é rotacionada em $90^{\circ}$ no sentido horário, e a cada interação, a peça rotaciona mais $90^{\circ}$. A fim de alterar a amplitude dos movimentos dos pacientes é utilizada uma variável de normalização, calculada para cada indivíduo. Desse modo, a intensidade do movimento pode ser ajustada, permitindo a interação independente das limitações apresentadas pelos pacientes.

A princípio, a interação está sendo realizada por meio do controle Gear VR, que possui sensores inerciais e botões. No entanto, uma nova forma de interação é desejada, onde o usuário, provido de Biomechamic Sensor Node (BSN), possa interagir com a aplicação por meio do rastreamento dos seus movimentos. O BSN é fruto da pesquisa do CEPID BRAINN em parceria com a spin off Bioxthica. A Figura 3 apresenta o $\mathrm{BSN}$, composto por acelerômetro, giroscópio e magnetômetro.

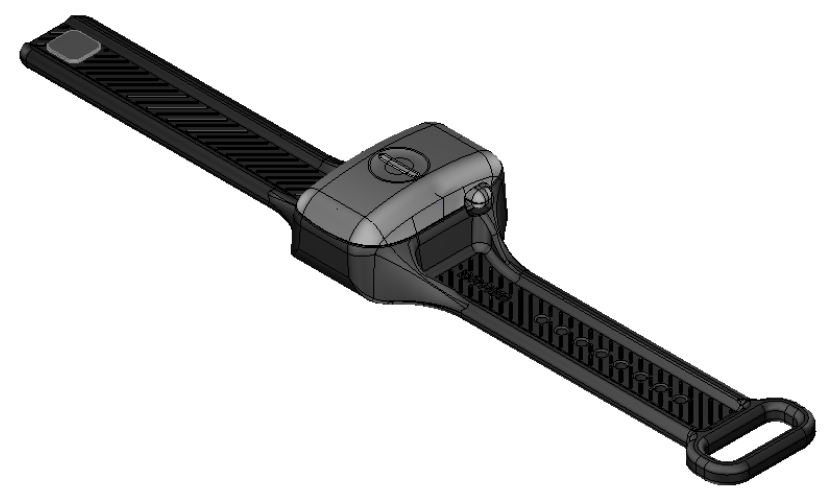

Figura 3. Biomechamic Sensor Node(BSN)

Ainda em relação à interação, existe a possibilidade do uso 
da Myo Armband, uma pulseira provida de sensores inerciais e também de um sensor de eletromiografia. A Figura 4 apresenta a Myo Armband, que possui proposta semelhante ao BSN.

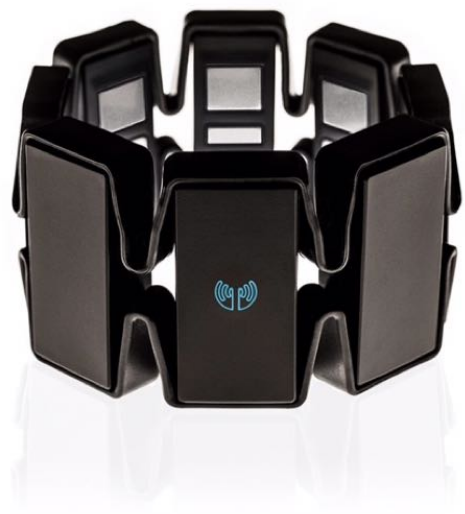

Figura 4. Myo Armband

A aplicação do profissional de saúde possibilita o acompanhamento das ações do paciente em outro dispositivo, smartphone ou computador pessoal. Deste modo, o profissional é capaz de iniciar, reiniciar e trocar as imagens. Ademais, também é possível rotacionar as peças do jogo, caso julgue necessário este tipo de auxílio ao paciente. A Figura 5 apresenta a interface da aplicação do profissional.

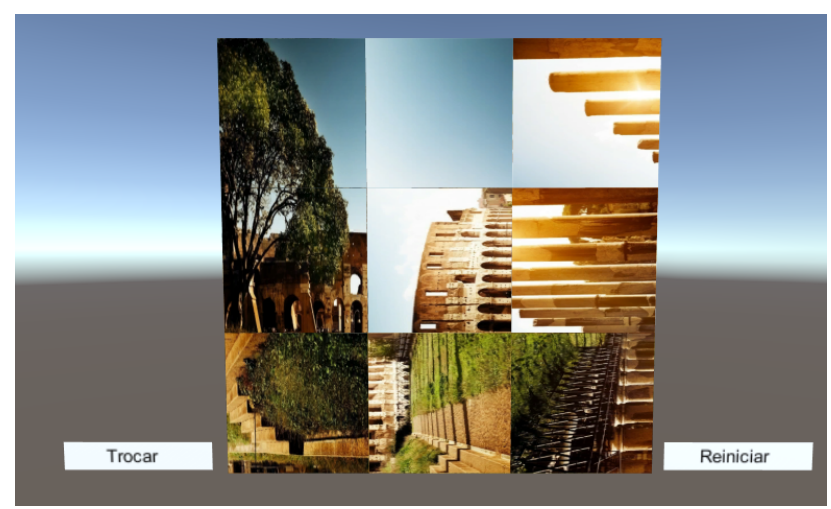

Figura 5. Interace da aplicação do profissional

\section{RESULTADOS PRELIMINARES}

A pesquisa ainda está em condução, uma vez que a aplicação foi finalizada no início de julho. A próxima etapa é realizar a avaliação da aplicação junto ao Brazilian Institute of Neuroscience and Neurotechnology (BRAINN).

Em relação às questões tecnológicas, alguns testes já foram realizados. O ambiente já foi exportado para as plataformas Android e Windows, permitindo que o paciente visualize a aplicação em um dispositivo Android compatível com o GearVR; já o profissional, pode utilizar um dispositivo Android ou um computador pessoal para interagir com o ambiente.
Há ainda o que se discutir em relação à interação, uma vez que questões referentes à restrição de movimentos do paciente devem ser consideradas. Por exemplo, com o GearVR é possível rastrear os movimentos do paciente por meio dos sensores inerciais e rotacionar as peças mediante ao clique de um botão do dispositivo. Já no caso do BSN, os movimentos podem ser rastreados devido aos sensores inerciais, mas a seleção das peças não pode ser feita por meio de botões, resultando em uma questão a ser solucionada. Por fim, no que se refere à Myo Armband, o sensor de eletromiografia poderia ser uma solução em relação à seleção das peças, mas os movimentos reconhecidos pela pulseira devem ser bem definidos, algo difícil de ser realizado por um paciente com limitações em seus movimentos.

\section{CONClusões}

O presente trabalho apresenta a proposta de uma aplicação voltada à reabilitação, ainda na UTI, de pacientes que sofreram um AVC. Espera-se contribuir para verificação da hipótese de pesquisa que considera as primeiras horas de um paciente pósAVC influentes em sua reabilitação

A solução imersiva já foi escolhida e validada. No entanto, em relação à interação, algumas questões ainda estão em fase de testes. Atualmente, a solução baseada em Myo Armband está sendo testada, com intuito de que pequenos estímulos realizados pelos dedos do paciente possam ser reconhecidos como input à aplicação. A próxima etapa do projeto consiste em realizar o piloto com pacientes dentro do Hospital de Clínicas da Unicamp.

\section{REFERÊNCIAS}

[1] Michael Brainin and Richard D Zorowitz. Advances in stroke: recovery and rehabilitation. Stroke, 44(2):311-313, 2013.

[2] Bermúdez I Badia S. Duarte E. Verschure P. F Cameirão, M. S. Virtual reality based rehabilitation speeds up functional recovery of the upper extremities after stroke: a randomized controlled pilot study in the acute phase of stroke using the rehabilitation gaming system. Restorative Neurology and Neuroscience, 29(5), 287- 298, 29(5):287-298, 2011.

[3] Bo Ryun Kim, Min Ho Chun, Lee Suk Kim, and Ji Young Park. Effect of virtual reality on cognition in stroke patients. Annals of rehabilitation medicine, 35(4):450, 2011.

[4] Anat Mirelman, Benjamin L Patritti, Paolo Bonato, and Judith E Deutsch. Effects of virtual reality training on gait biomechanics of individuals poststroke. Gait \& posture, 31(4):433-437, 2010.

[5] Pfizer. O que é o acidente vascular cerebral, quais os tipos, como prevenir e tratar. https://www.pfizer.com.br/noticias/O-que-e-acidente-vascularcerebral-AVC-tipos-prevencao-tratamento, 2019. [Online; Acessado em 29 de julho de 2019].

[6] José Eduardo Pompeu, Thais Helena Alonso, Igor Bordello Masson, Sandra Maria Alvarenga Anti Pompeu, and Camila Torriani-Pasin. Os efeitos da realidade virtual na reabilitação do acidente vascular encefálico: Uma revisão sistemática. Motricidade, 10(4):111-122, 2014.

[7] Silvia Cristina Sawada. Fatores associados ao AVC entre idosos do município de São Paulo-resultados do estudo SABE. PhD thesis, Universidade de São Paulo.

[8] Emmanuel Tsekleves, Ioannis Theoklitos Paraskevopoulos, Alyson Warland, and Cherry Kilbride. Development and preliminary evaluation of a novel low cost vr-based upper limb stroke rehabilitation platform using wii technology. Disability and Rehabilitation: Assistive Technology, 11(5):413-422, 2016. 